\title{
Does immune checkpoint inhibitor increase the risks of poor outcomes in COVID-19-infected cancer patients? A systematic review and meta-analysis
}

\author{
Gilbert Lazarus $^{1}$ (D) Refael Alfa Budiman ${ }^{1} \cdot$ Ikhwan Rinaldi $^{2}$
}

Received: 26 September 2020 / Accepted: 16 June 2021 / Published online: 26 June 2021

(c) The Author(s), under exclusive licence to Springer-Verlag GmbH Germany, part of Springer Nature 2021

\begin{abstract}
Background The association between immune checkpoint inhibitor (ICI) and outcomes of cancer patients with coronavirus disease 2019 (COVID-19) infection has yet to be systematically evaluated. This meta-analysis aims to investigate the effects of ICI treatment on COVID-19 prognosis, including mortality, severity, and any other prognosis-related outcomes.

Methods Eligible studies published up to 27 February 2021 were included and assessed for risk of bias using the Quality in Prognosis Studies tool. A random-effects meta-analysis was conducted to estimate the pooled effect size along with its 95\% confidence intervals. The quality of body evidence was evaluated using the modified Grading of Recommendations Assessment, Development, and Evaluation framework.

Results Eleven studies involving a total of 2826 COVID-19-infected cancer patients were included in the systematic review. We discovered a moderate-to-high quality of evidence that ICI was not associated with a higher mortality risk, while the other outcomes yielded a very low-to-low-evidence quality. Although our findings indicated that ICI did not result in a higher risk of severity and hospitalization, further evidence is required to confirm our findings. In addition, we discovered that prior exposure to chemoimmunotherapy may be linked with a higher risk of COVID-19 severity (OR 8.19 [95\% CI: 2.67-25.08]; $I^{2}=0 \%$, albeit with small sample size.

Conclusion Our findings indicated that ICI treatment should not be adjourned nor terminated during the current pandemic. Rather, COVID-19 vigilance should be increased in such patients. Further studies with larger cohorts and higher quality of evidence are required to substantiate our findings.
\end{abstract}

Trial registration number This project has been prospectively registered at PROSPERO (registration ID: CRD42020202142) on 4 August 2020.

Keywords Checkpoint inhibitor · COVID-19 · Neoplasms · Prognosis · Programmed cell death 1 receptor

\section{Abbreviations}

AKI Acute kidney injury

ARDS Acute respiratory distress syndrome

CENTRAL Cochrane controlled register of trials

CINAHL Cumulative index to nursing and allied health literature

Gilbert Lazarus

gilbert.lazarus@ui.ac.id

1 Faculty of Medicine, Universitas Indonesia, Jl. Salemba Raya No. 6, RW 5, Kenari, Kec. Senen, Kota Jakarta Pusat, Jakarta 10430, Indonesia

2 Division of Hematology and Medical Oncology, Department of Internal Medicine, Faculty of Medicine, Universitas Indonesia - Cipto Mangunkusumo Hospital, Jakarta, Indonesia
COVID-19 Coronavirus disease 2019

CKD Chronic kidney disease

COPD Chronic obstructive pulmonary disease

CTLA-4 Cytotoxic T-lymphocyte associated protein 4

CVD Cardiovascular disease

DIC Disseminated intravascular coagulation

ECOG Eastern cooperative oncology group

GRADE Grading of recommendations assessment, development and evaluation

ICI Immune checkpoint inhibitor

ICU Intensive care unit

PD-1 Programmed cell death protein 1

PD-L1 Programmed death ligand 1

QUIPS Quality in prognosis studies tool

WHO World health organization 


\section{Introduction}

The current coronavirus disease 2019 (COVID-19) pandemic has brought upon a significant burden in the global economy and health, resulting in millions of cases and nearly one million of death [1]. Recent reports have suggested that cancer patients are more vulnerable to COVID-19-related deaths and complications[2-4]; thus, meticulous management to prevent further deterioration in such patients is essential. In light of this, the question to whether postpone or continue active cancer treatments, including immune checkpoint inhibitor (ICI) which exerts immunomodulatory functions [5], remains. To the best of our knowledge, the current evidence on the effect of prior ICI treatment on cancer patients infected with COVID-19 remains contentious [6-8]. Therefore, this meta-analysis aims to explore the association between ICI and COVID-19 outcomes in cancer patients, thus providing the best available evidence to guide real-time treatment decisions in such patients.

\section{Methods}

This systematic review adhered to the guideline of systematic review of prognostic factor studies by Riley et al.[9] and was reported according to the Preferred Reporting Items for Systematic Reviews and Meta-Analyses statement[10]. A detailed protocol has been registered prospectively at PROSPERO (CRD42020202142[11]).

\section{Search strategy and selection criteria}

We conducted a comprehensive search on PubMed, Scopus, MEDLINE (via EBSCO), Cumulative Index to Nursing and Allied Health Literature (CINAHL), Cochrane Controlled Register of Trials (CENTRAL), and the World Health Organization (WHO) COVID-19 research databases, searching for relevant studies published from inception up to 27 February 2021 with keywords listed in Supplementary Table S1. Furthermore, we also searched grey literature (i.e. Google Scholar, ProQuest, MedRxiv, BioRxiv, and Social Science Research Network) databases, in addition to manually hand-searching the reference lists of the included studies and similar reviews. Lastly, we retrieved similar records of the included studies with the PubMed's 'similar articles' algorithm and subsequently deduplicated and screened them against the pre-specified eligibility criteria. No language restrictions were applied during the search.

Literature searches were performed by two independent investigators, with any discrepancies resolved by the blind assessment of a third investigator. The retrieved records were screened against the following inclusion criteria: (1) study design, primary studies including case series or letter to editors with at least 10 patients; (2) population and exposure, studies enrolling COVID-19-infected cancer patients with and without prior exposure to ICIs; and (3) outcomes, including mortality, severity, and any other prognosis-related outcomes. Due to heterogeneity of reporting, we conformed to the authors' definition of prior ICI exposure and severity endpoint. In the case of studies only mentioning immunotherapy as an exposure to COVID-19 patients, the corresponding authors were contacted to confirm their study settings, and the studies were subsequently excluded when there was no response (see Additional methods in the Supplementary Material for more details). Contrariwise, records were excluded if the full-text articles were non-English or irretrievable.

\section{Data extraction and risk-of-bias assessment}

The following information was extracted from each included studies: (1) author and year of publication; (2) study characteristics, including recruitment period, study design, settings, location and sample size; (3) patient characteristics, including age, proportion of male patients, comorbidities, cancer types, adjuvant therapies, and characteristics of ICI, i.e., time to last ICI exposure and type of ICI; and (4) outcomes. The primary outcomes in this review were the risk of mortality and severity among COVID-19-infected cancer patients. Whenever possible, outcomes were further investigated per criterion according to the WHO interim guidance, viz., rate of hospitalization, intensive care unit (ICU) admission, invasive ventilation, acute respiratory distress syndrome (ARDS), and shock [12]. Data extraction was performed by one review author (GL) using a pre-specified sheet in MS Excel ${ }^{\circledR}$ for Office 365 MSO ver. 2002 (Microsoft Corporation, Redmond, WA, 2018). A second investigator (RAB) checked the accuracy of the extracted data, and any disagreements were resolved by the consensus between the authors.

Any reported effect size types (hazard ratio [HR], odds ratio [OR], relative risk $[R R]$ ) were incorporated in this study. When only binary data were provided, unadjusted ORs were calculated from the frequency of events and sample sizes [13]. Furthermore, when ICI was split into multiple groups (i.e., ICI monotherapy and ICI plus chemotherapy), the within-study groups were combined into a single pairwise comparison using a fixed-effect model as recommended by Cochrane [14]. In the case of studies reporting multiple adjustment sets, we extracted the adjusted set incorporating the greatest number of covariates.

The included studies were further assessed for risk of bias by using the Quality in Prognosis Studies (QUIPS) tool [15], where the overall risk of bias was judged to be 
low, moderate, and high. Risk-of-bias assessments were conducted by two independent reviewers, and any discrepancies were resolved by a third adjudicator in a blinded fashion. Details on the QUIPS checklist can be found in Supplementary Table S2.

\section{Data analysis and synthesis}

Data analyses were performed by using the $\mathrm{R}$ ver. 4.0.0 (R Foundation for Statistical Computing, Vienna, Austria) [16] with the additional meta package [17]. In the case of studies with overlapping populations, analyses were prioritized to the largest-sized study. Outcomes were pooled as ORs, RRs, or HRs separately along with their $95 \%$ confidence intervals (CIs) by using the generic inverse variance model. Both unadjusted and adjusted outcomes were extracted and synthesized in this study; however, adjusted estimates were prioritized for reporting and interpretation whenever available. Due to the likeliness of unexplained heterogeneity [9], a DerSimonian-Laird random-effects model was used [18]. Heterogeneity between studies was investigated with Cochran's $Q$ test and $I^{2}$ statistics. According to $\mathrm{I}^{2}$ values, heterogeneity was classified as negligible (0-25\%), low (25-50\%), moderate (50-75\%), or high $(>75 \%)$, while the significance level for Q statistics were set at $10 \%$.

A priori, we defined subgroups according to study design, location, sample size, and risk of bias, while additional subsets based on the presence of adjuvant therapy (ICI monotherapy and ICI plus chemotherapy), cancer type (lung and non-lung cancer), and comparator groups (no active treatment, chemotherapy, targeted therapy, radiotherapy, and surgery) were determined posteriori. A priori-determined subgroup analyses were performed only for outcomes with at least two studies in at least two subsets. On the other hand, sensitivity analysis was conducted by excluding studies with high risk of bias and simultaneously performing leave-one-out analyses. When the number of studies was adequate $(n \geq 10)$ [19], potential publication bias was investigated by the visual inspection of contour-enhanced funnel plots [20] and the quantitative analysis with Egger's[21] and Begg's tests [22].

Lastly, the overall quality of evidence was assessed with the modified version of the Grading of Recommendations Assessment, Development and Evaluation (GRADE) framework for prognostic reviews [23], where the certainty of evidence was rated as high, moderate, low, or very low according to the judgments of these following domains: phase of investigation, study limitation, inconsistency, indirectness, imprecision, publication bias, moderate/large effect sizes, and exposure-response gradients.

\section{Results}

\section{Search results and study characteristics}

The initial search yielded 1948 records, of which 776 were deduplicated and 1112 were excluded following title and abstract screening. The remaining 60 studies were retrieved for full-text assessments, where 27 studies were excluded due to inappropriate design (24 case reports/series with $<10$ patients and 3 commentaries), 16 due to inappropriate settings (nine studies only included ICI-exposed COVID-19 patients, four studies with non-ICI immunotherapy, two studies investigating non-COVID-19 viral infections, and one study with unidentifiable setting; see Additional methods in the Supplementary Materials for further details), and five ongoing studies (three trial records and two study protocols).. Consequently, 11 studies with a total of 2826 patients were included in this review-among which 1510 (53.4\%) were male, and hypertension was the most common comorbidity (40.3\%; Table 1). Lastly, we expanded our search by using a nonhuman skill-dependent search method based on PubMed's 'similar articles' algorithm, in addition to manually handsearching the reference lists of included studies. No new studies were identified from these expanded searches. Details on the literature search strategy are illustrated on Fig. 1. Among the included studies, five were conducted in Europe [24-28], four in America [29-32], and one each in Asia [33] and multiple regions [34]. All but one [26] study were conducted retrospectively, and most were multicentered (seven out of 11). Most patients suffered from solid tumor (2195 [77.7\%]), and nearly half of the cases were metastatic (1217 [43.1\%]). Among them, the most frequent cancer type was lung cancer $(19.9 \%)$, followed by gastrointestinal (14.8\%) and breast tumors (13.2\%). With regard to ICI type, most patients received anti-PD-1 (4.3\%), followed by anti-PD-L1 (2.0\%) and anti-CTLA-4 (1.7\%; Table 1).

Risk-of-bias assessments resulted in low risk for five studies [24, 26, 29, 30, 34] and moderate [31, 33] and high risk $[25,28,32]$ for three studies each. Most of the included studies yielded moderate-to-high risks in the study attrition and confounding domains (Supplementary Fig S1), which may be explained by the fact that all but one study [26] were done retrospectively. Furthermore, four studies reported that their findings might potentially be limited by the small sample sizes [25, 30, 32, 33], thus further signifying the potential biases. Details on the risk-of-bias assessment for each signaling question can be found in Supplementary Fig S2. 


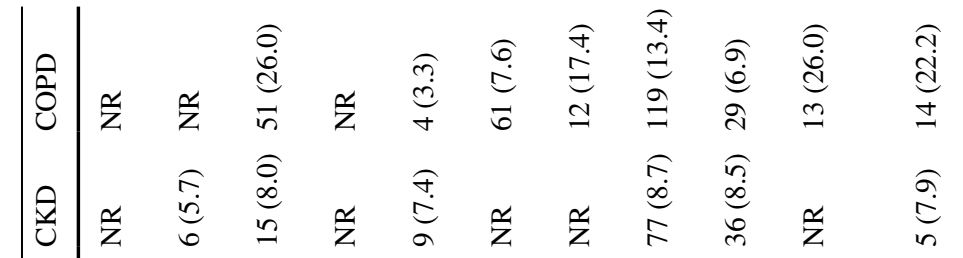

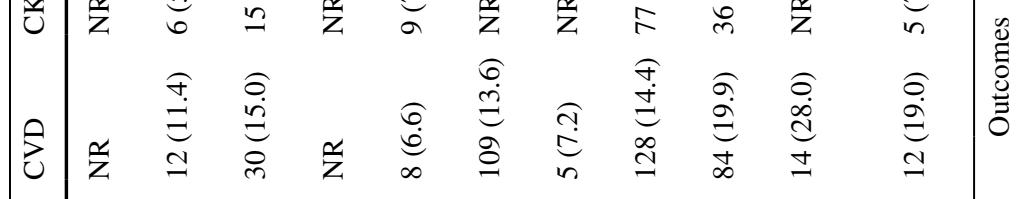

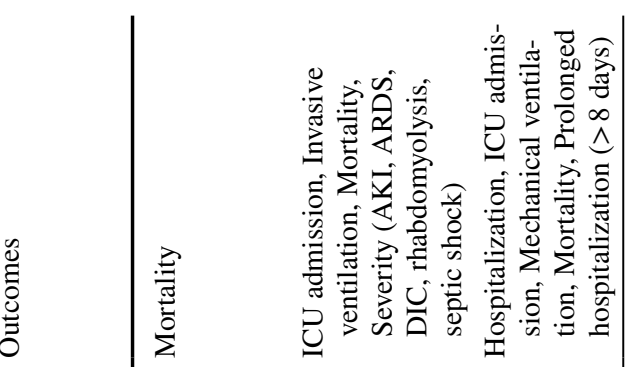

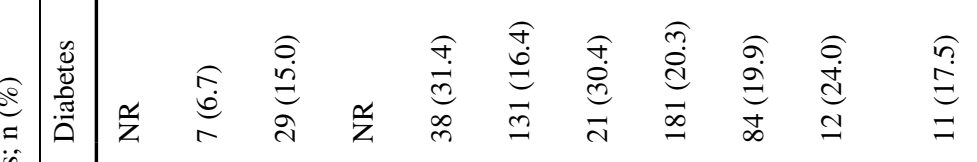

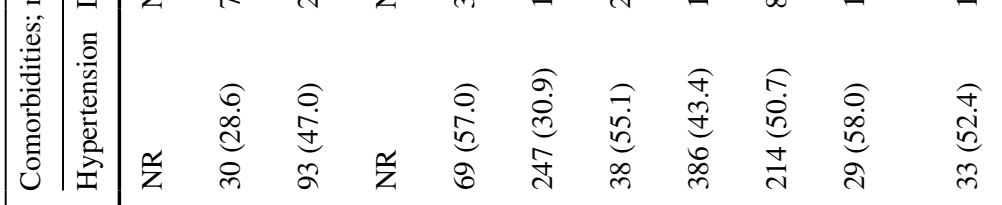

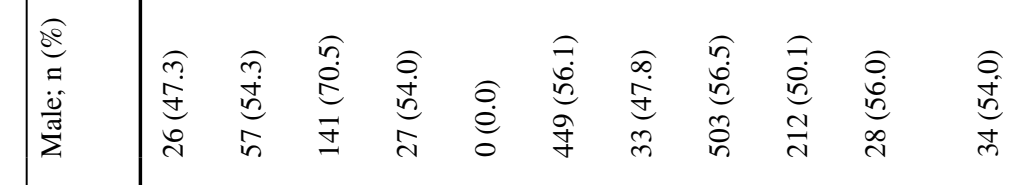

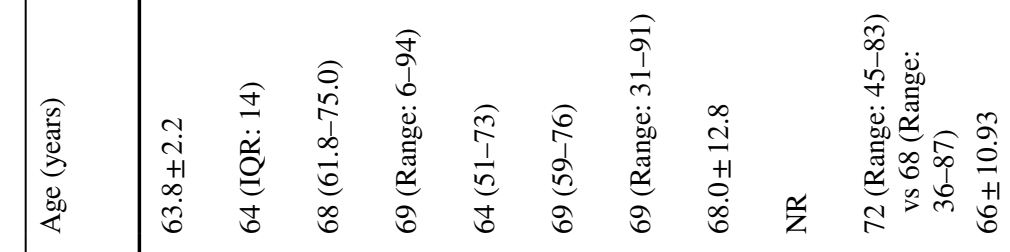

.

in

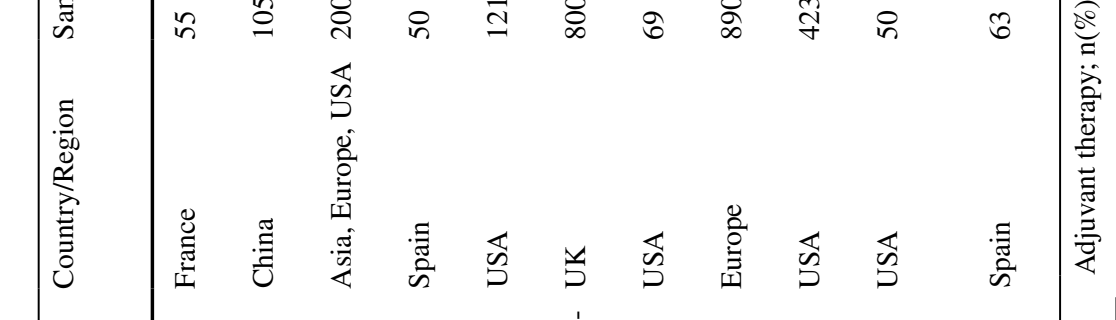

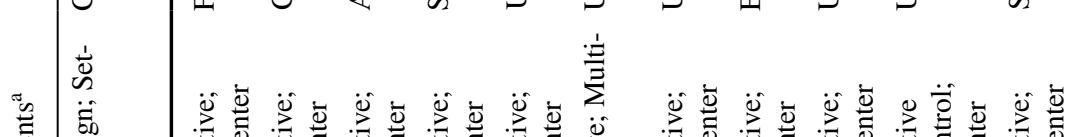

窝

营

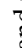

龸

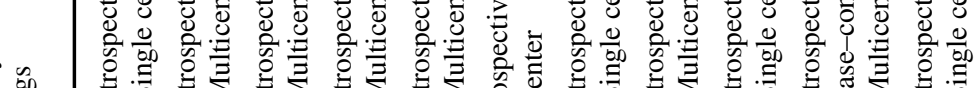

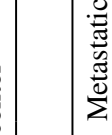

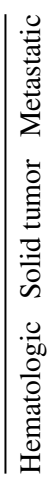

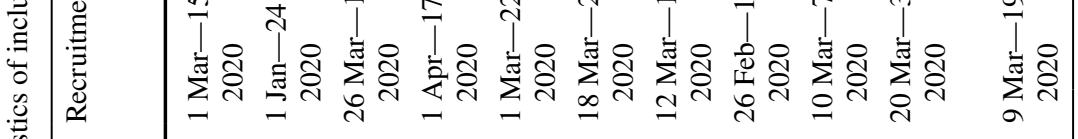

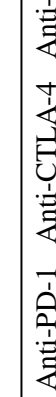

安

$\stackrel{0}{i}$

艺

事

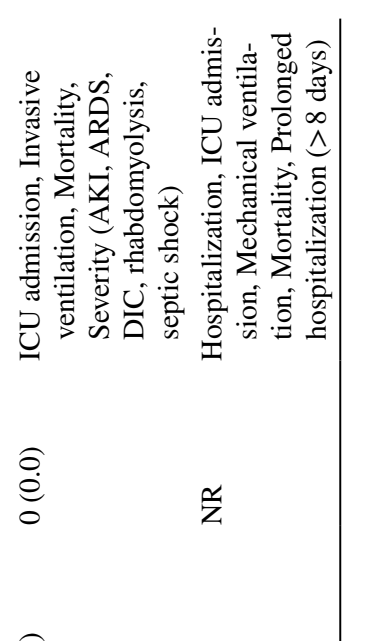

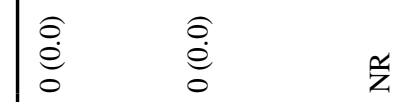

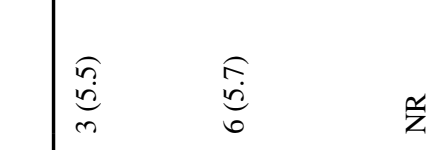

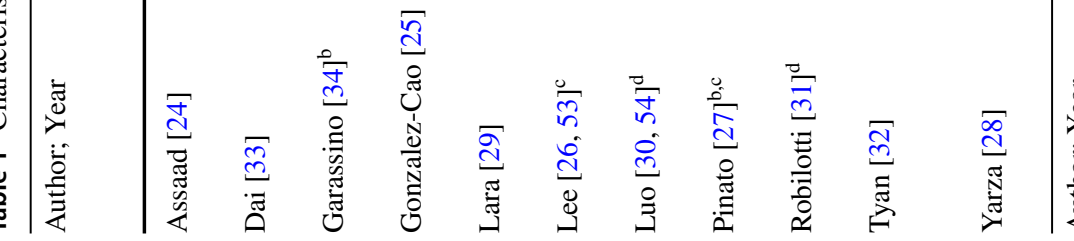

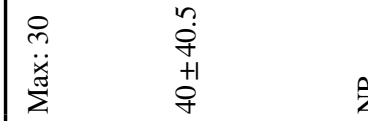

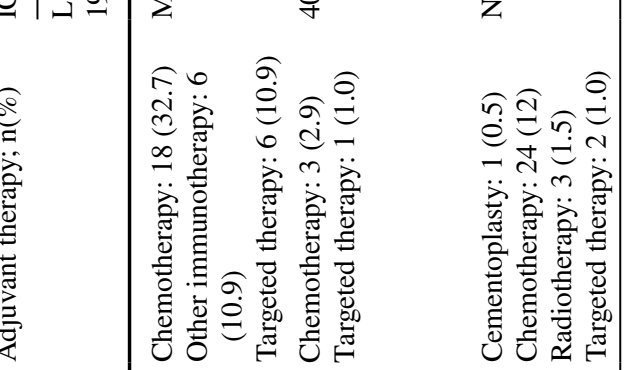

Z

党

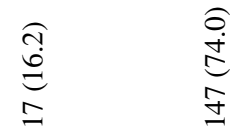

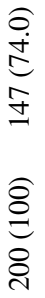

$\stackrel{\varrho}{\stackrel{0}{0}}$

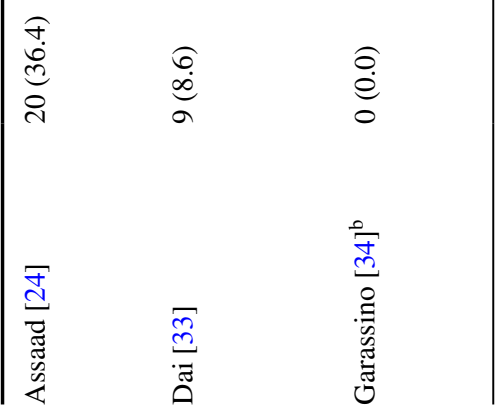




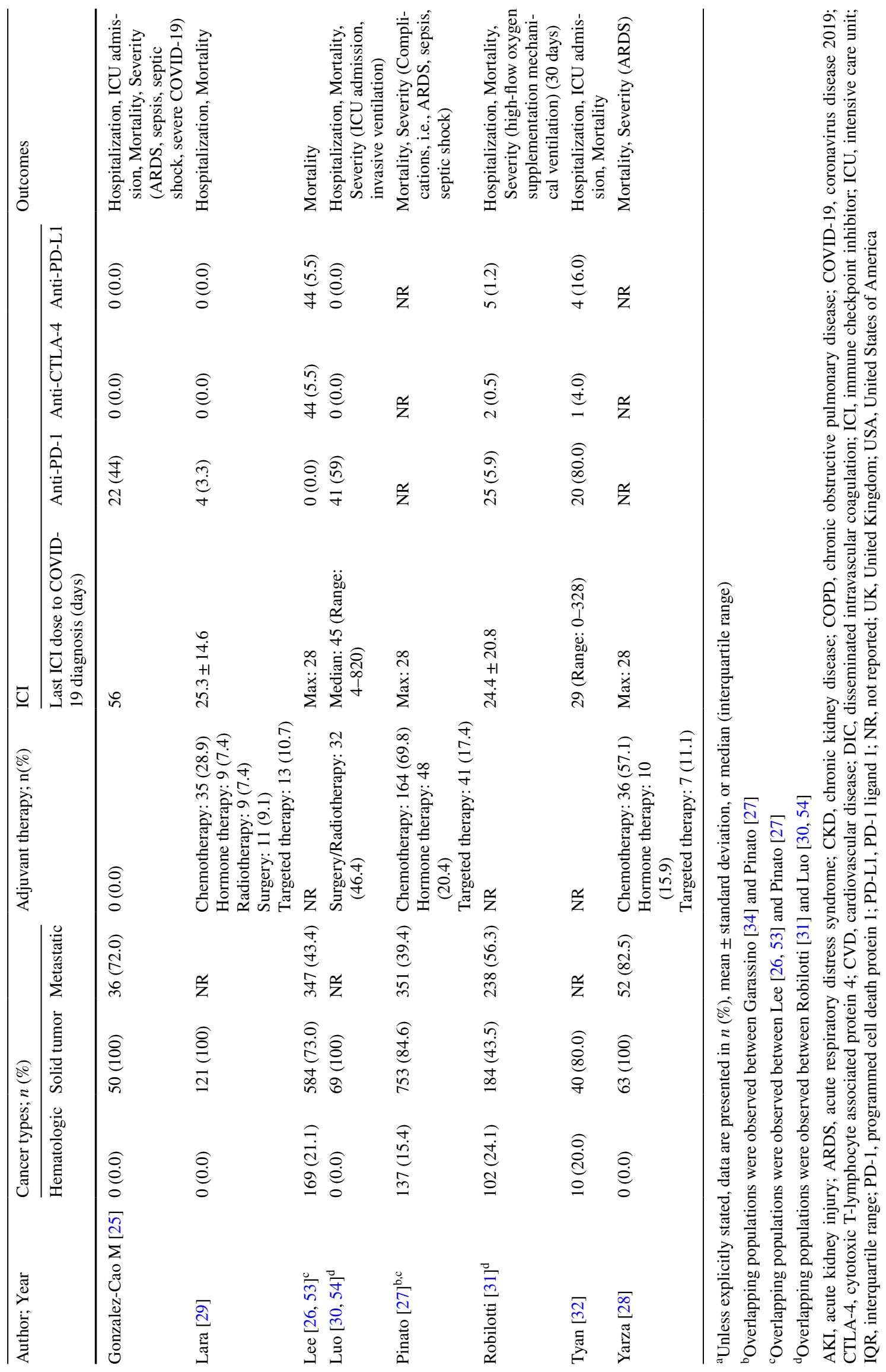




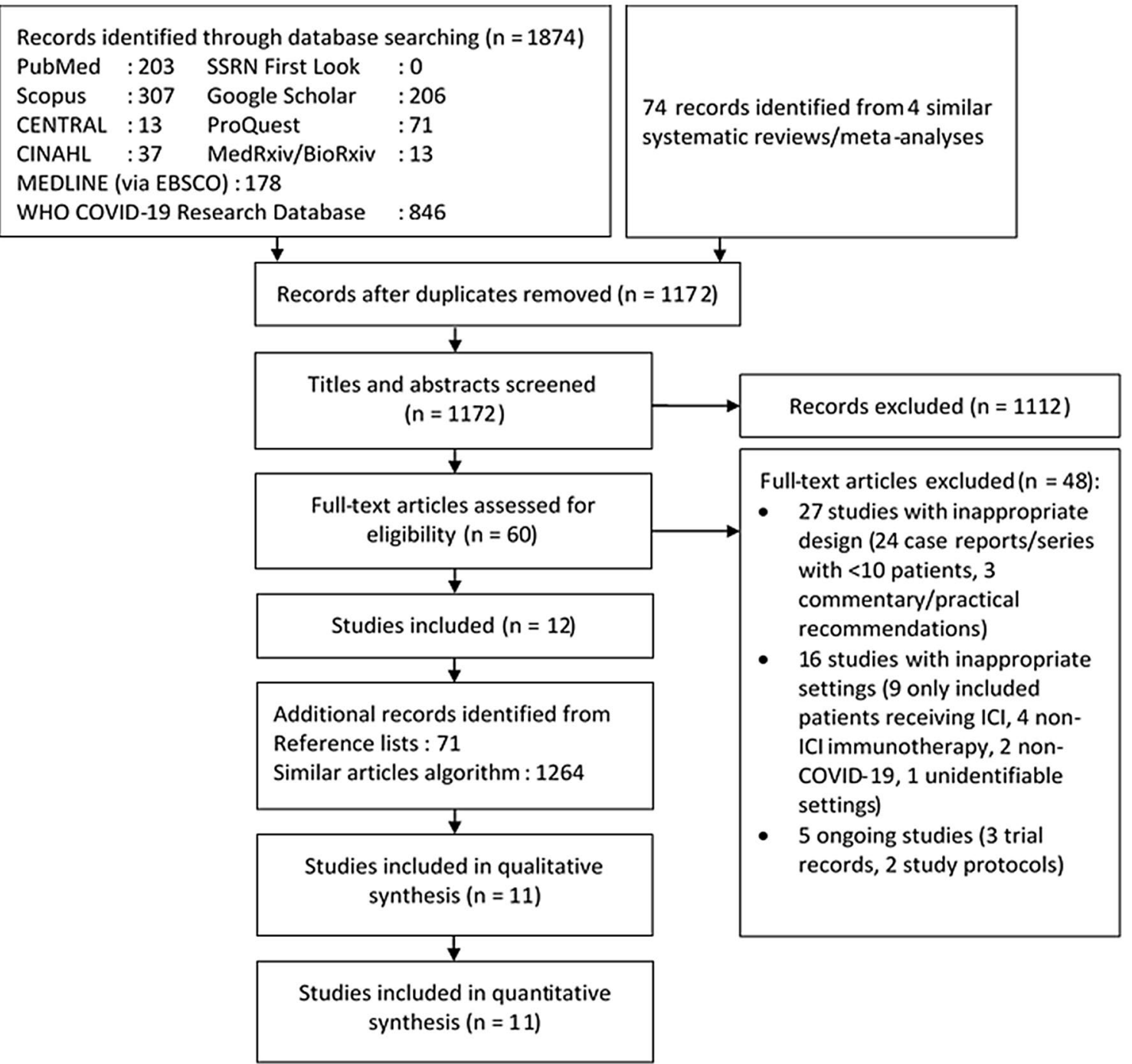

Fig. 1 Diagram flow illustrating the literature search strategy. CENTRAL, Cochrane Central Register of Controlled Trials; CINAHL, Cumulative Index to Nursing and Allied Health Literature; SSRN, Social Science Research Network; WHO, World Health Organization

\section{Outcomes}

The summary of pooled unadjusted and adjusted effects can be found in Table 2, while the certainty of evidence according to GRADE assessment can be seen on Supplementary Table S3. GRADE assessments of the qualitative and quantitative analysis on the effects of prior ICI treatment on COVID-19 mortality resulted in moderate- and high-evidence quality, respectively, while the remaining outcomes yielded very low-to-low quality of evidence. Publication bias assessments were not performed as no outcomes yielded more than 10 studies [35].

Our findings suggested that prior exposure to ICI was not associated with COVID-19 mortality (OR 0.91 [95\% CI: 0.61-1.38]; Fig. 2a), which was supported by our findings from the analysis of the adjusted outcomes (OR $0.70[95 \%$
CI: 0.40-1.23]; Fig. 3a)—both with negligible heterogeneity $\left(I^{2}=2 \%\right.$ and $I^{2}=0 \%$, respectively; both $\left.\mathrm{p}>0.10\right)$. We also found that studies with moderate-to-high risk of bias tend to yield wider CIs and higher heterogeneity. Nonetheless, we showed that these studies did not contribute much to the overall estimates as our findings remained relatively robust following sensitivity analyses (Supplementary Figure S3AB). Considering this, we judged the certainty of evidence for the quantitative assessment as high, while that of the qualitative assessment was judged as moderate. Subgroup analyses based on cancer type, presence of adjuvant therapy, and comparator group also revealed similar trends, thus further ascertaining our findings.

Similar to mortality, we also observed a non-significant association between prior ICI treatment with severity and hospitalization (OR 1.47 [95\% CI: 0.95-2.27], $I^{2}=5 \%$, 
Table 2 Pooled adjusted and unadjusted effects of prior ICI exposure on COVID-19 outcomes

\begin{tabular}{|c|c|c|c|c|c|c|}
\hline \multirow[t]{2}{*}{ Outcome } & \multirow[t]{2}{*}{ Studies } & \multicolumn{2}{|c|}{ Events/N } & \multirow[t]{2}{*}{ OR $(95 \%$ CI $)$} & \multicolumn{2}{|c|}{ Heterogeneity } \\
\hline & & ICI & No ICI & & $I^{2}$ & $P$-value \\
\hline \multicolumn{7}{|l|}{ Adjusted effects } \\
\hline Mortality ${ }^{\mathrm{a}, \mathrm{b}}$ & $5[26,28,30,32,33]$ & $30 / 122^{c}$ & $237 / 963^{c}$ & $0.70(0.40-1.23)$ & $0 \%$ & 0.606 \\
\hline \multicolumn{7}{|l|}{ Subgroup analysis } \\
\hline \multicolumn{7}{|l|}{ Sample size } \\
\hline$<100$ patients & $3[28,30,32]$ & $18 / 72^{\mathrm{c}}$ & $12 / 108^{c}$ & $0.71(0.29-1.73)$ & $0 \%$ & 0.595 \\
\hline$\geq 100$ patients & $2[26,33]$ & $12 / 50$ & $223 / 855$ & $0.90(0.22-3.69)$ & $40 \%$ & 0.195 \\
\hline \multicolumn{7}{|l|}{ Risk of bias } \\
\hline Low & $2[26,30]$ & $21 / 83$ & $221 / 784$ & $0.68(0.34-1.35)$ & $0 \%$ & 0.451 \\
\hline Moderate/High & $3[28,32,33]$ & $9 / 39^{c}$ & $16 / 179^{c}$ & $0.75(0.27-2.15)$ & $6 \%$ & 0.346 \\
\hline \multicolumn{7}{|l|}{ Location } \\
\hline Asia & $1[33]$ & $2 / 6$ & $7 / 99$ & $3.03(0.29-31.98)$ & NA & NA \\
\hline Europe & $2[26,28]$ & $10 / 52^{\mathrm{c}}$ & $216 / 811^{\mathrm{c}}$ & $0.63(0.31-1.25)$ & $0 \%$ & 0.749 \\
\hline America & $2[30,32]$ & $18 / 64$ & $14 / 53$ & $0.67(0.22-2.05)$ & $2 \%$ & 0.314 \\
\hline \multicolumn{7}{|l|}{ Adjuvant therapy } \\
\hline ICI monotherapy & $1[28]$ & NR & NR & $0.15(0.01-1.65)$ & NA & NA \\
\hline $\begin{array}{l}\text { ICI+ chemo- } \\
\text { therapy }\end{array}$ & $1[28]$ & NR & NR & $1.96(0.29-13.18)$ & NA & NA \\
\hline Severity & $3[30,33]$ & $19 / 45$ & $132 / 546$ & $1.62(0.48-5.43)$ & $57 \%$ & 0.095 \\
\hline \multicolumn{7}{|l|}{ Subgroup analysis } \\
\hline \multicolumn{7}{|l|}{ Adjuvant therapy } \\
\hline ICI monotherapy & $1[28]$ & NR & NR & $0.26(0.03-1.88)$ & NA & NA \\
\hline $\begin{array}{l}\text { ICI + chemo- } \\
\text { therapy }\end{array}$ & $1[28]$ & NR & NR & $0.97(0.14-6.45)$ & NA & NA \\
\hline Hospitalization $^{\mathrm{d}, \mathrm{e}}$ & $1[31]$ & $18 / 29$ & $150 / 382$ & $2.84(1.22-6.72)$ & NA & NA \\
\hline \multicolumn{7}{|l|}{ Unadjusted effects } \\
\hline Mortality $^{\mathrm{b}}$ & 8 [24-26, 29, 30, 32-34] & $51 / 198$ & $317 / 1241$ & $0.91(0.60-1.38)$ & $2 \%$ & 0.411 \\
\hline \multicolumn{7}{|l|}{ Subgroup analysis } \\
\hline \multicolumn{7}{|l|}{ Sample size } \\
\hline$<100$ patients & $4[24,25,30,32]$ & $21 / 89$ & $28 / 133$ & $0.95(0.46-1.94)$ & $0 \%$ & 0.609 \\
\hline$\geq 100$ patients & $4[26,29,33,34]$ & $30 / 109$ & $289 / 1108$ & $1.05(0.51-2.18)$ & $44 \%$ & 0.150 \\
\hline \multicolumn{7}{|l|}{ Risk of bias } \\
\hline Low & $5[24,26,29,30,34]$ & $39 / 145$ & 295/1089 & $0.89(0.56-1.41)$ & $0 \%$ & 0.450 \\
\hline Moderate/High & $3[25,32,33]$ & $12 / 53$ & $22 / 152$ & $1.06(0.34-3.25)$ & $42 \%$ & 0.178 \\
\hline \multicolumn{7}{|l|}{ Location } \\
\hline Asia & $1[33]$ & $2 / 6$ & $7 / 99$ & $4.45(0.72-27.44)$ & NA & NA \\
\hline Europe & 3 [24-26] & $13 / 69$ & $13 / 836$ & $0.60(0.30-1.22)$ & $0 \%$ & 0.989 \\
\hline America & $3[29,30,32]$ & $20 / 71$ & $30 / 167$ & $1.30(0.61-2.78)$ & $0 \%$ & 0.389 \\
\hline International & $1[34]$ & $16 / 52$ & $50 / 139$ & $0.79(0.40-1.57)$ & NA & NA \\
\hline \multicolumn{7}{|l|}{ Cancer type } \\
\hline Lung cancer & $3[30,33,34]$ & $28 / 96$ & $58 / 184$ & $0.98(0.55-1.74)$ & $0 \%$ & 0.495 \\
\hline $\begin{array}{l}\text { Non-lung solid } \\
\text { cancer }\end{array}$ & $3[25,29,33]$ & $6 / 30$ & $26 / 215$ & $4.00(0.30-52.88)$ & $87 \%$ & $<0.001$ \\
\hline \multicolumn{7}{|l|}{ Adjuvant therapy } \\
\hline ICI monotherapy & $4[25,29,33,34]$ & $16 / 60$ & $128 / 380$ & $0.88(0.43-1.81)$ & $6 \%$ & 0.364 \\
\hline $\begin{array}{l}\text { ICI + chemo- } \\
\text { therapy }\end{array}$ & $3[29,33,34]$ & $7 / 23$ & $76 / 352$ & $1.12(0.34-3.70)$ & $46 \%$ & 0.159 \\
\hline \multicolumn{7}{|l|}{ Comparator group ${ }^{\mathrm{f}}$} \\
\hline No treatment & $5[24-26,33,34]$ & $32 / 127$ & $120 / 421$ & $0.86(0.42-1.77)$ & $34 \%$ & 0.193 \\
\hline Chemotherapy $^{\mathrm{g}}$ & $5[25,26,29,33,34]$ & $33 / 131$ & $106 / 334$ & $0.83(0.46-1.51)$ & $16 \%$ & 0.310 \\
\hline Targeted therapy & $5[24,26,29,33,34]$ & $30 / 112$ & $26 / 120$ & $1.19(0.63-2.22)$ & $0 \%$ & 0.753 \\
\hline
\end{tabular}


Table 2 (continued)

\begin{tabular}{|c|c|c|c|c|c|c|}
\hline \multirow[t]{2}{*}{ Outcome } & \multirow[t]{2}{*}{ Studies } & \multicolumn{2}{|c|}{ Events/N } & \multirow[t]{2}{*}{ OR $(95 \% \mathrm{CI})$} & \multicolumn{2}{|c|}{ Heterogeneity } \\
\hline & & ICI & No ICI & & $I^{2}$ & $P$-value \\
\hline Surgery & $3[26,29,33]$ & $14 / 57$ & $11 / 48$ & $1.11(0.45-2.77)$ & $0 \%$ & 0.840 \\
\hline Radiotherapy & $3[26,29,33]$ & $15 / 57$ & $20 / 98$ & $2.03(0.48-8.66)$ & $36 \%$ & 0.212 \\
\hline Hormone therapy & $2[26,29]$ & $13 / 51$ & $22 / 73$ & $1.43(0.12-16.47)$ & $59 \%$ & 0.117 \\
\hline Severity ${ }^{\mathrm{b}, \mathrm{d}, \mathrm{h}, \mathrm{i}}$ & $6[25,27-29,31,33]$ & $72 / 130$ & $699 / 1522$ & $1.47(0.95-2.27)$ & $5 \%$ & 0.384 \\
\hline \multicolumn{7}{|l|}{ Subgroup analysis } \\
\hline \multicolumn{7}{|l|}{ Sample size } \\
\hline$<100$ patients & $2[25,28]$ & $18 / 30$ & $54 / 100$ & $1.99(0.50-7.86)$ & $2 \%$ & 0.313 \\
\hline$\geq 100$ patients & $4[27,29,31,33]$ & $41 / 83$ & $658 / 1439$ & $1.40(0.82-2.40)$ & $26 \%$ & 0.258 \\
\hline \multicolumn{7}{|l|}{ Location } \\
\hline Asia & $1[33]$ & $4 / 6$ & $36 / 99$ & $3.50(0.61-20.06)$ & NA & NA \\
\hline Europe & $3[25,27,28]$ & $55 / 86$ & $569 / 917$ & $1.05(0.61-1.78)$ & $0 \%$ & 0.925 \\
\hline America & $2[29,31]$ & $13 / 38$ & $94 / 506$ & $2.35(1.14-4.83)$ & $0 \%$ & 0.322 \\
\hline \multicolumn{7}{|l|}{ Cancer type } \\
\hline Lung cancer ${ }^{\mathrm{j}}$ & $2[30,33]$ & $18 / 44$ & $17 / 43$ & $1.27(0.51-3.19)$ & $0 \%$ & 0.758 \\
\hline $\begin{array}{l}\text { Non-lung solid } \\
\text { cancer }\end{array}$ & $4[25,29,31,33]$ & $22 / 49$ & $97 / 445$ & $1.49(0.72-3.07)$ & $0 \%$ & 0.407 \\
\hline \multicolumn{7}{|l|}{ Adjuvant therapy } \\
\hline ICI monotherapy & $4[25,29,31,33]$ & $22 / 44$ & $175 / 633$ & $1.25(0.56-2.79)$ & $0 \%$ & 0.579 \\
\hline $\begin{array}{l}\text { ICI + chemo- } \\
\text { therapy }\end{array}$ & $3[29,31,33]$ & $10 / 15$ & $112 / 605$ & $8.72(3.03-25.11)$ & $0 \%$ & 0.703 \\
\hline \multicolumn{7}{|l|}{ Comparator group $^{f}$} \\
\hline No treatment & $3[25,31,33]$ & $31 / 59$ & $68 / 285$ & $2.39(1.24-4.62)$ & $0 \%$ & 0.490 \\
\hline Chemotherapy & $5[25,28,29,31,33]$ & $35 / 74$ & $60 / 230$ & $1.75(0.84-3.67)$ & $0 \%$ & 0.592 \\
\hline Targeted therapy & $3[28,31,33]$ & $19 / 45$ & $19 / 77$ & $2.17(0.95-4.93)$ & $0 \%$ & 0.499 \\
\hline Surgery & $2[29,33]$ & $5 / 13$ & $7 / 19$ & $0.99(0.18-5.35)$ & $0 \%$ & 0.788 \\
\hline Radiotherapy & $2[29,33]$ & $6 / 13$ & $4 / 22$ & $5.91(0.98-35.71)$ & $0 \%$ & 0.836 \\
\hline Hormone therapy & $2[28,29]$ & $5 / 15$ & $5 / 19$ & $1.33(0.25-6.98)$ & $0 \%$ & 0.421 \\
\hline Hospitalization $^{\mathrm{b}, \mathrm{d}, \mathrm{i}}$ & $5[25,29,31,32,34]$ & $99 / 137$ & $368 / 694$ & $1.04(0.49-2.22)$ & $53 \%$ & 0.076 \\
\hline \multicolumn{7}{|l|}{ Subgroup analysis } \\
\hline \multicolumn{7}{|l|}{ Sample size } \\
\hline$<100$ patients & $2[25,32]$ & $35 / 47$ & $47 / 53$ & $0.36(0.09-1.40)$ & $21 \%$ & 0.261 \\
\hline$\geq 100$ patients & $3[29,31,34]$ & $64 / 90$ & $321 / 641$ & $1.60(0.92-2.79)$ & $12 \%$ & 0.321 \\
\hline \multicolumn{7}{|l|}{ Risk of bias } \\
\hline Low & $2[29,34]$ & $46 / 61$ & $171 / 259$ & $1.15(0.59-2.25)$ & $0 \%$ & 0.970 \\
\hline Moderate/High & $3[25,31,32]$ & $53 / 76$ & $197 / 435$ & $0.74(0.15-3.65)$ & $76 \%$ & 0.016 \\
\hline \multicolumn{7}{|l|}{ Cancer type } \\
\hline Lung cancer ${ }^{\mathrm{j}}$ & $2[30,34]$ & $69 / 94$ & $172 / 193$ & $1.32(0.72-2.39)$ & $0 \%$ & 0.570 \\
\hline $\begin{array}{l}\text { Non-lung solid } \\
\text { cancer }\end{array}$ & $3[25,29,31]$ & $28 / 46$ & $167 / 358$ & $1.07(0.52-2.17)$ & $0 \%$ & 0.559 \\
\hline \multicolumn{7}{|l|}{ Adjuvant therapy } \\
\hline ICI monotherapy & $4[25,29,31,34]$ & $51 / 74$ & $344 / 669$ & $1.06(0.59-1.89)$ & $0 \%$ & 0.772 \\
\hline $\begin{array}{l}\text { ICI + chemo- } \\
\text { therapy }\end{array}$ & $3[29,31,34]$ & $26 / 31$ & $321 / 641$ & $2.10(0.37-12.03)$ & $62 \%$ & 0.073 \\
\hline \multicolumn{7}{|l|}{ Comparator group ${ }^{f}$} \\
\hline No treatment & $3[25,31,34]$ & $76 / 105$ & $135 / 269$ & $1.25(0.46-3.40)$ & $61 \%$ & 0.075 \\
\hline Chemotherapy & $4[25,29,31,34]$ & $80 / 112$ & $112 / 223$ & $1.49(0.66-3.33)$ & $42 \%$ & 0.159 \\
\hline Targeted therapy & $3[29,31,34]$ & $64 / 90$ & $52 / 124$ & $2.54(1.37-4.72)$ & $0 \%$ & 0.919 \\
\hline Surgery & $1[29]$ & $5 / 7$ & $5 / 11$ & $3.00(0.40-22.71)$ & NA & NA \\
\hline Radiotherapy & $1[29]$ & $5 / 7$ & $4 / 9$ & $3.13(0.38-25.57)$ & NA & NA \\
\hline Hormone therapy & $1[29]$ & $5 / 7$ & $4 / 9$ & $3.13(0.38-25.57)$ & NA & NA \\
\hline
\end{tabular}


Table 2 (continued)

\begin{tabular}{|c|c|c|c|c|c|c|}
\hline \multirow[t]{2}{*}{ Outcome } & \multirow[t]{2}{*}{ Studies } & \multicolumn{2}{|c|}{ Events/N } & \multirow[t]{2}{*}{ OR $(95 \% \mathrm{CI})$} & \multicolumn{2}{|c|}{ Heterogeneity } \\
\hline & & ICI & No ICI & & $I^{2}$ & $P$-value \\
\hline $\mathrm{ICU}_{\text {admission }}{ }^{\mathrm{e}}$ & $2[25,32]$ & $6 / 47$ & $13 / 53$ & $0.38(0.12-1.16)$ & $0 \%$ & 0.967 \\
\hline $\begin{array}{l}\text { Prolonged hospitaliza- } \\
\text { tion }(>8 \text { days })^{\mathrm{e}}\end{array}$ & $1[34]$ & $10 / 17$ & $21 / 41$ & $1.36(0.43-4.27)$ & NA & NA \\
\hline \multicolumn{7}{|l|}{ Subgroup analysis } \\
\hline \multicolumn{7}{|l|}{ Adjuvant therapy } \\
\hline $\begin{array}{l}\text { ICI + chemo- } \\
\text { therapy }\end{array}$ & $1[34]$ & $3 / 7$ & $21 / 41$ & $0.71(0.14-3.60)$ & NA & NA \\
\hline
\end{tabular}

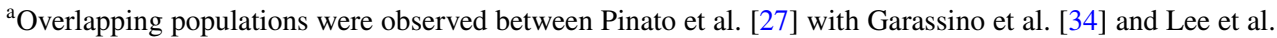
[26], of which Pinato et al.[27] was excluded due to smaller cumulative sample size

${ }^{\mathrm{b}}$ Subgroup analysis based on study design was not performed due to paucity of studies ( $<2$ subsets with $\geq 2$ studies)

${ }^{\mathrm{c}}$ The event rate may be underestimated as Yarza et al.[28] did not provide the number of deaths among patients receiving and not receiving ICI

${ }^{\mathrm{d}}$ Overlapping populations were observed between Luo et al.[30] and Robilotti et al.[31], of which Robilotti[31] et al. was prioritized due to larger sample size

${ }^{\mathrm{e}} \mathrm{A}$ priori-determined subgroup and sensitivity analysis was not performed due to paucity of studies

${ }^{\mathrm{f}}$ For study-specific estimates, see Supplementary Table S5

${ }^{\mathrm{g}}$ Assaad et al. was excluded as both arms had no events[24]

${ }^{\mathrm{h}}$ Subgroup analysis based on risk of bias was not performed due to paucity of studies ( $<2$ subsets with $\geq 2$ studies)

${ }^{\text {i }}$ Subgroup analysis based on study location was not performed due to paucity of studies ( $<2$ subsets with $\geq 2$ studies)

${ }^{j}$ Overlapping lung cancer patients were observed between Luo et al.[30] and Robilotti et al.[31], of which Luo et al.[30] was prioritized due to larger sample size. CI, confidence interval; ICI, immune checkpoint inhibitor; ICU, intensive care unit; OR, odds ratio

$p=0.384 ;$ and OR 1.04 [95\% CI: $0.49-2.22$ ] $I^{2}=53 \%$, $p=0.076$; respectively; Fig. $2 \mathrm{~b}-\mathrm{c})$. Subgroup analysis revealed that the moderate heterogeneity observed in the hospitalization model was derived from studies with moderate-to-high risk of bias $\left(I^{2}=76 \%, p=0.016\right)$. However, we were unable to establish a firm evidence as the non-significant association of the severity and hospitalization outcomes shifted towards right following the exclusion of Pinato et al. [27] in the severity model and Garassino et al. [34] in the hospitalization model (Supplementary Figure S3C-D). Furthermore, analysis of the adjusted outcomes revealed a higher risk of hospitalization among ICI-exposed patients (Table 2), while those of severity outcome remained nonsignificant (OR 1.62 [95\% CI: 0.48-5.43]; Fig. 3b), although with moderate heterogeneity $\left(I^{2}=57 \%, p=0.095\right)$. These indicated that further evidence is required to confirm our findings as most of the current findings were still equivocal. Considering this, we judged the quality of evidence on the qualitative assessments of COVID-19 severity and hospitalization to be low, and those of quantitative assessments to be very low. In addition, preliminary evidence also showed that prior ICI exposure did not result in a higher risk of ICU admission (OR 0.38 [95\% CI: 0.12-1.16], $I^{2}=0 \%, p=0.967$; Fig. 2d). However, as both studies included in the model yielded high risk of bias[25, 32], further studies are required to substantiate these results.

Subset analyses based on cancer type and the presence of adjuvant therapy for hospitalization outcome revealed similar trends to those of mortality outcome. Nonetheless, we found that concomitant use of ICI and chemotherapy was associated with a higher risk of COVID-19 severity (OR 8.19 [2.67-25.08]; $I^{2}=0 \%, p=0.441$ ), although Yarza et al. stated that the association between ICI exposure and COVID-19 severity was non-significant (OR 0.97 [95\% CI: 0.14-6.45])—independent of age, sex, metastatic cancer, chronic obstructive pulmonary disease (COPD), history of venous thromboembolism, and Eastern Cooperative Oncology Group (ECOG) performance status (Supplementary Table S4). Furthermore, we also found that the risk of severity was higher in ICI-treated patients than patients with no active cancer treatment (OR 2.39 [95\% CI: 1.24-4.62], $\left.I^{2}=0 \%\right)$. Nonetheless, it is important to note that the observed effects were primarily driven by a single study[31] as the other studies $[25,33]$ yielded small sample sizes and wide CIs (Supplementary Table S5). 
(A) Mortality

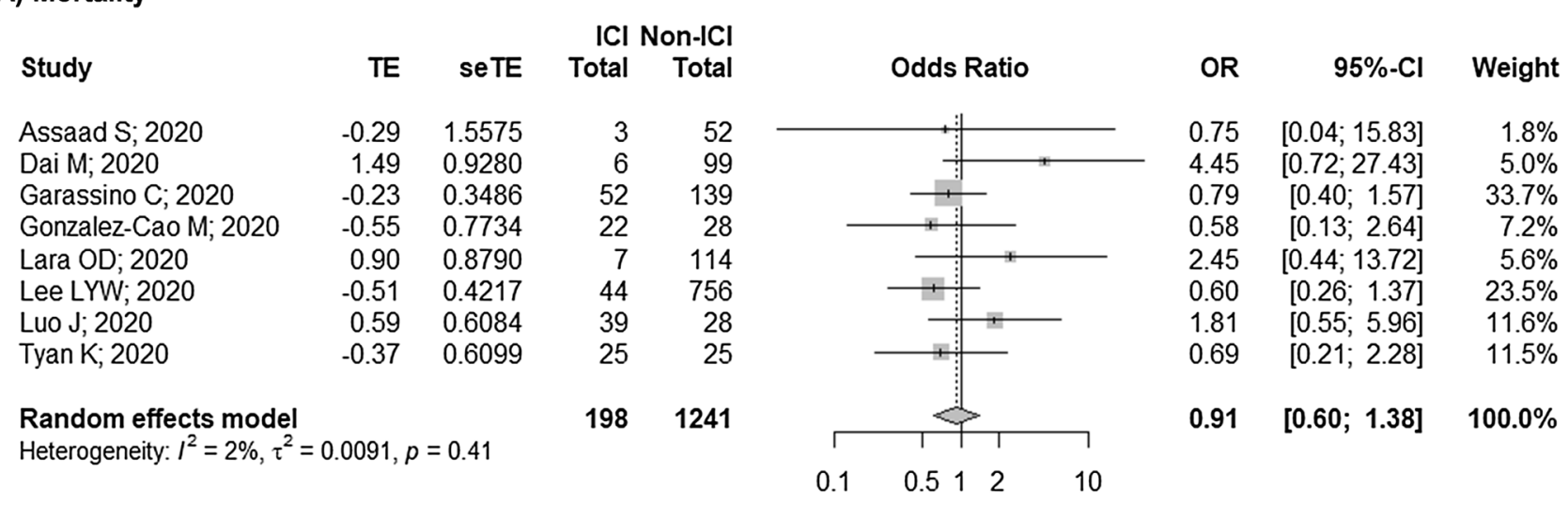

(B) Severity ${ }^{a}$

$\begin{array}{lrrrr}\text { Study } & \text { TE } & \text { se TE } & \text { Total } & \text { Total } \\ & & & & \\ \text { Dai M; 2020 } & 1.25 & 0.8909 & 6 & 99 \\ \text { Lara OD; 2020 } & -0.18 & 1.1090 & 7 & 114 \\ \text { Gonzalez-Cao M; 2020 } & -0.15 & 0.6201 & 22 & 28 \\ \text { Pinato DJ; 2020 } & 0.11 & 0.3287 & 56 & 834 \\ \text { Robilotti EV; 2020 } & 0.98 & 0.3905 & 31 & 392 \\ \text { Yarza R; 2020 } & -0.03 & 0.7813 & 8 & 55 \\ & & & 130 & 1522\end{array}$

(C) Hospitalization ${ }^{a}$

\begin{tabular}{lrrrr} 
& & & \multicolumn{2}{c}{ ICI Non-ICl } \\
Study & TE & seTE & Total & Total \\
Garassino C; 2020 & 0.14 & 0.3796 & 54 & 145 \\
Gonzalez-Cao M; 2020 & -0.55 & 0.6875 & 22 & 28 \\
Lara OD; 2020 & 0.11 & 0.7866 & 7 & 114 \\
Robilotti EV; 2020 & 0.93 & 0.3968 & 29 & 382 \\
Tyan K; 2020 & -2.03 & 1.1229 & 25 & 25 \\
& & & & \\
Random effects model & & & 137 & 694 \\
Heterogeneity: $I^{2}=53 \%, \tau^{2}=0.3604, p=0.08$ & & &
\end{tabular}

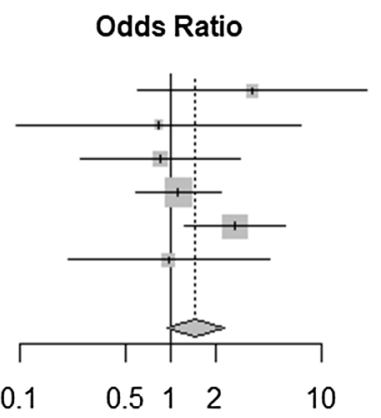

$\begin{array}{rrr}\text { OR } & 95 \%-C l & \text { Weight } \\ & & \\ 3.50 & {[0.61 ; 20.06]} & 6.2 \% \\ 0.83 & {[0.09 ; 7.32]} & 4.0 \% \\ 0.86 & {[0.25 ; 2.89]} & 12.4 \% \\ 1.12 & {[0.59 ; 2.13]} & 40.0 \% \\ 2.67 & {[1.24 ; 5.74]} & 29.5 \% \\ 0.97 & {[0.21 ; 4.49]} & 8.0 \% \\ & & \\ 1.47 & {[0.95 ; 2.27]} & 100.0 \%\end{array}$

(D) ICU admission

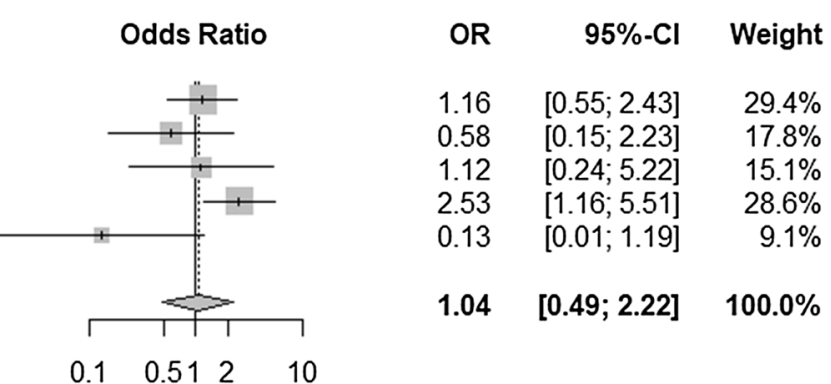

ICI Non-ICl

$\begin{array}{lrrrr}\text { Study } & \text { TE } & \text { seTE } & \text { Total } & \text { Total } \\ \text { Gonzales-Cao; } 2020 & -0.92 & 1.1920 & 22 & 28 \\ \text { Tyan K; 2020 } & -0.98 & 0.6455 & 25 & 25 \\ & & & 47 & 53 \\ \text { Random effects model } & & & 47\end{array}$

Fig. 2 Pooled unadjusted estimates on the association between prior ICI exposure with risks of: a mortality, $\mathbf{b}$ severity, $\mathbf{c}$ hospitalization, and $\mathbf{d}$ ICU admission. ICI, immune checkpoint inhibitor. ICU, inten-

\section{Discussion}

This meta-analysis showed that prior exposure to ICI was not associated with poorer prognosis in COVID-19-infected cancer patients. We demonstrated that there was a

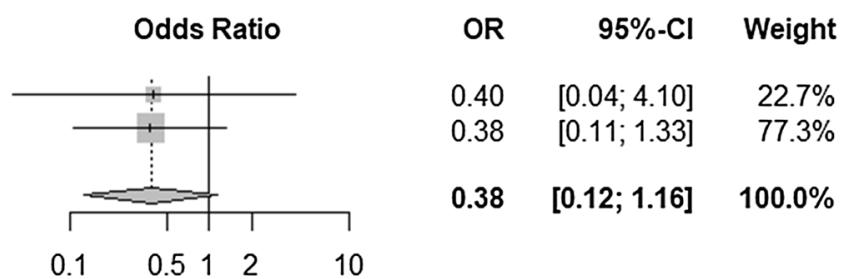

sive care unit. ${ }^{a}$ Overlapping populations were observed between Luo et al. [30] and Robilotti et al. [31], of which Robilotti [31] et al. was prioritized due to larger sample size

moderate-to-high strength of evidence that ICI did not result in a higher risk of COVID-19 mortality, while the certainty of evidence for other outcomes yielded very low-to-low quality - which is quite expected considering that most of the included studies yielded a moderate-to-high risk of bias. 


\begin{tabular}{|c|c|c|c|c|}
\hline \multirow[b]{2}{*}{ Study } & \multirow[b]{2}{*}{ TE } & \multirow[b]{2}{*}{ seTE } & \multicolumn{2}{|c|}{$\mathrm{ICI}$ Non-ICl } \\
\hline & & & Total & Tota \\
\hline Dai M; 2020 & 1.11 & 1.2016 & 6 & 99 \\
\hline Lee LYW; 2020 & -0.53 & 0.3950 & 44 & 756 \\
\hline Luo J; 2020 & 0.12 & 0.7657 & 39 & 28 \\
\hline Tyan K; 2020 & -1.02 & 0.8381 & 25 & 25 \\
\hline Yarza R; $2020^{\mathrm{b}}$ & -0.25 & 0.7798 & 8 & 55 \\
\hline $\begin{array}{l}\text { Random effec } \\
\text { Heterogeneity: } I^{2}\end{array}$ & 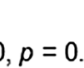 & & 122 & 963 \\
\hline
\end{tabular}

\section{(B) Severityc}

\begin{tabular}{lrrrr} 
& & & \multicolumn{2}{c}{ ICI Non-ICl } \\
Study & TE & seTE & Total & Total \\
& & & & \\
Dai M; 2020 & 0.70 & 1.0440 & 6 & 99 \\
Robilotti EV; 2020 & 1.18 & 0.4361 & 31 & 392 \\
Yarza R; 2020' & -0.64 & 0.7170 & 8 & 55 \\
& & & & \\
Random effects model & & & 45 & 546
\end{tabular}

Fig. 3 Pooled adjusted estimates on the association between prior ICI exposure with risks of a mortality and $\mathbf{b}$ severity. ICI, immune checkpoint inhibitor. a Overlapping populations were observed between Pinato et al. [27] with Garassino et al. [34] and Lee et al. [26], of
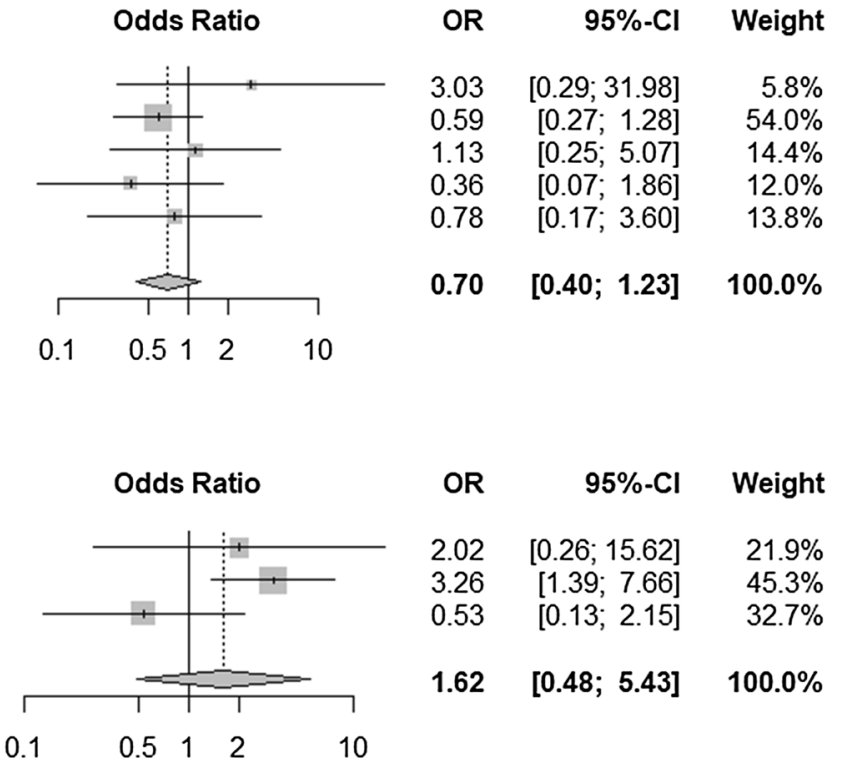

which Pinato et al. [27]. ' Effect size was derived by combining multiple groups into a single pair-wise comparison. ${ }^{\mathrm{c} O v e r l a p p i n g ~ p o p u l a-}$ tions were observed between Luo et al. [30] and Robilotti et al. [31], of which Robilotti [31] et al. was prioritized due to larger sample size

confounders, and considering that the preliminary findings by Yarza et al. suggested otherwise [28], further evidence is required to confirm these findings.

The dilemma to whether continue, postpone, or even terminate active cancer treatment, including ICI, remained relevant during the current COVID-19 pandemic. While physicians are expected to prioritize patients' safety, it is also important to ensure that the patients receive timely treatments. Several reports and guidelines have regarded ICI as unsafe during the pandemic, and have advised the postponement of such treatments due to safety considerations [41-43]. These are based on two hypothetical adverse interactions between ICI and COVID-19 infection. First, recent reports have suggested that COVID-19 infection may mask ICI-related pneumonitis symptoms, thus potentially delaying essential treatments $[7,40]$. Although this might be detrimental considering that ICI-related pneumonitis accounts for about one-third of treatment-related deaths in cancer patients, their incidence is relatively rare. Furthermore, the risks of ICI pneumonitis tend to be augmented in early ICI recipients and super-responders [44], suggesting that a prompt and accurate risk stratification, in addition to an increased COVID-19 vigilance, may be able to mitigate this issue.

In addition, early hypotheses postulated that ICI may worsen COVID-19 outcomes due to potential immune [40]. However, as the model was unadjusted by potential 
hyperactivation [44-46], where they may upregulate proinflammatory cytokines [44, 45] and over-activate CD8 T-cells[46] - resulting in the dysregulation and exhaustion of T-cells [44, 47]. This hypothesis was supported by the fact that severe COVID-19 cases were associated with lymphopenia and immune hyperactivity [6, 45], thus suggesting that ICI may synergistically exacerbate cytokine storm in COVID-19 infection [46]. Nevertheless, a recent report by Di Cosimo et al. stated that the occurrence of cytokine storm in COVID-19 patients was more likely to be driven by direct viral damage rather than immune-mediated inflammation [48]. Moreover, recent studies have argued the potential role of ICI on the prevention and management of COVID19 infection. ICI has exhibited immunity protection against several infectious agents [45], while also restoring cellularmediated immunocompetence resulting in increased viral control $[6,49]$. In addition, ICI may also enhance immune response to viral antigens without triggering adverse immune reactions [48], thus further suggesting the potential therapeutic utility of ICI.

Altogether, these findings indicated that ICI treatment should not be unnecessarily deferred during the current pandemic; but rather, COVID-19 vigilance in ICI-treated cancer patients should be increased. This is especially important to ensure prompt diagnosis and treatment of COVID-19 infections, thus preventing adverse outcomes in such patients. The decision to continue or suspend ICI treatment should be based on case-by-case approaches $[44,50]$, where treatment adjustments may be performed to mitigate the risk of COVID-19 infection by reducing patients' contacts to medical system, rather than due to ICI-related safety concerns. This is saliently important considering that cancer patients receiving active anticancer therapy may be at an increased COVID-19 infection risk due to frequent visits to hospitals [51]. Furthermore, specific approaches to certain populations may be adopted, e.g., early treatment discontinuation in patients with complete or prolonged response[8], adjustments of treatment intervals or modality [7,52], or adjournments of ICI therapy in high risk patients (e.g., elderly, patients with history of immune-related adverse events and/ or comorbidities) [52].

This study has several limitations. Although our findings rejected the early hypotheses stating that ICI may cause deleterious effects to COVID-19-infected cancer patients, study paucity and small-sized cohorts limited the interpretation of our results. Furthermore, some models (severity, hospitalization, and ICU admission) were also limited by the predominant studies with moderate-to-high-bias risk, which was further worsened by the observed heterogeneity in hospitalization outcome, thus resulting in the judgment of very low-to-low-evidence quality. Nonetheless, we demonstrated a moderate-to-high quality of evidence that ICI was not associated with COVID-19 mortality. Moreover, although most of the included studies were conducted retrospectively, the studies involved diverse populations, thus ascertaining the generalizability of our findings. Despite this, it should be noted that none of the included studies directly compared the risks between different ICI classes, implying that further studies with larger ICI cohorts are required to confirm our findings and to explore the observed effects.

In addition, due to heterogeneity of reporting, we were unable to ascertain the association between the proximity of last ICI exposure to COVID-19 outcomes, thus indicating that future studies should aim to explore the potential effect of this variable. Although preliminary evidence suggested that this association was non-significant [30], such a finding was derived from a relatively small sample size, hence suggesting that future studies with larger sample sizes are required to substantiate this finding. Furthermore, we also recommend future studies to specifically investigate the association between prior ICI exposure and COVID-19 outcomes in hematological malignancies and in patients receiving chemoimmunotherapy as the current evidence is still inconclusive. Lastly, although our eligibility criteria may introduce language bias, we did not discover any potentially eligible non-English article during the literature search process, thereby suggesting that any potential bias was insignificant.

To the best of our knowledge, this is the first meta-analysis conducted to evaluate the association between prior ICI exposure and COVID-19 outcomes. Although our findings were limited due to study scarcity and small-sized cohorts, we were able to establish a moderate-to-high certainty of evidence on the non-significant relationship between ICI and COVID-19 mortality. We hope that our findings may encourage physicians to increase COVID-19 vigilance among cancer patients and to perform risk-benefit assessments on each ICI-treated cancer patient rather than indiscriminately deferring ICI treatment, which may cause significant harms to cancer patients in the long run.

\section{Conclusion}

In conclusion, our findings suggested that prior ICI exposure was not associated with a higher risk of COVID-19 mortality in cancer patients, although future studies with larger cohorts and higher quality of evidence are required to confirm our findings on the association between ICI with COVID-19 severity and hospitalization. In light of this, we recommend that the adjournment of ICI treatments during the pandemic is unwarranted; but rather, COVID-19 vigilance on ICI-treated cancer patients should be performed more rigorously to ensure the early diagnosis and prompt 
management of such patients to prevent the occurrence of poor COVID-19 outcomes.

Supplementary Information The online version contains supplementary material available at https://doi.org/10.1007/s00262-021-02990-9.

Acknowledgements The authors would like to express their gratitude to Dr. Ying Taur and Dr. Mini Kamboj (Infectious Diseases, Department of Medicine, Memorial Sloan Kettering Cancer Center, New York, NY, USA) for the provision of additional data for analyses. The authors would also like to thank Dr. Lennard YW Lee (Institute of Cancer and Genomic Sciences, University of Birmingham, Edgbaston, Birmingham B15 2TT, UK) and Prof. Jeremy L. Warner (Vanderbilt-Ingram Cancer Center at Vanderbilt University Medical Center, Nashville, TN, USA) for the confirmation of study settings. Lastly, the authors thank Jessica Audrey (Faculty of Medicine, Universitas Indonesia, Jakarta, Indonesia) for the helpful insights which improved the manuscript.

Author's contribution GL conceptualized the idea, designed the methodology, administered the study protocol, undertook the formal analysis, and visualized the findings. GL, RAB, and IR screened the literature and assessed the risk of bias. GL and RAB extracted the data and drafted the original manuscript, while GL and IR reviewed and edited the manuscript for final submission. IR supervised the project. All authors have approved of the final manuscript for publication.

Funding This project received no specific grant from any funding agency in the public, commercial, or not-for-profit sectors.

Availability of data and material All data generated or analyzed during this study are included in this published article [and its supplementary information files].

Code availability Not applicable.

\section{Declarations}

Conflict of interest The authors declare no conflict of interest.

Ethics approval Not applicable.

Consent to participate Not applicable.

Consent for publication Not applicable.

\section{References}

1. World Health Organization (2020) Coronavirus disease (COVID19): weekly epidemiological update, 14 September 2020. World Health Organization. https://apps.who.int/iris/handle/10665/ 334304. Accessed 15 Sept 2020

2. Ofori-Asenso R, Ogundipe O, Agyeman AA et al (2020) Cancer is associated with severe disease in COVID-19 patients: a systematic review and meta-analysis. Ecancermedicalscience 14:1047. https://doi.org/10.3332/ecancer.2020.1047

3. Giannakoulis VG, Papoutsi E, Siempos II (2020) Effect of cancer on clinical outcomes of patients With COVID-19: a meta-Analysis of patient data. JCO Glob Oncol 6:799-808. https://doi.org/10. 1200/GO.20.00225
4. ElGohary GM, Hashmi S, Styczynski J et al (2020) The risk and prognosis of COVID-19 infection in cancer patients: a systematic review and meta-analysis. Hematol Oncol Stem Cell Ther S1658-3876(20):30122-30129. https://doi.org/10.1016/j.hemonc. 2020.07.005

5. Lazarus G, Audrey J, Iskandar AWB (2019) Efficacy and safety profiles of programmed cell death-1/programmed cell death ligand-1 inhibitors in the treatment of triple-negative breast cancer: a comprehensive systematic review. Oncol Rev. https://doi. org/10.4081/oncol.2019.425

6. Vivarelli S, Falzone L, Grillo CM et al (2020) Cancer management during COVID-19 pandemic: is immune checkpoint inhibitors-based immunotherapy harmful or beneficial? Cancers (Basel) 12:2237. https://doi.org/10.3390/cancers 12082237

7. Sullivan RJ, Johnson DB, Rini BI et al (2020) COVID-19 and immune checkpoint inhibitors: initial considerations. J Immunother Cancer 8:e00933. https://doi.org/10.1136/ jitc-2020-000933

8. Davis AP, Boyer M, Lee JH, Kao SC (2020) COVID-19: the use of immunotherapy in metastatic lung cancer. Immunotherapy 12:545-548. https://doi.org/10.2217/imt-2020-0096

9. Riley RD, Moons KGM, Snell KIE et al (2019) A guide to systematic review and meta-analysis of prognostic factor studies. BMJ. https://doi.org/10.1136/bmj.k4597

10. Moher D, Liberati A, Tetzlaff J et al (2009) Preferred reporting items for systematic reviews and meta-analyses: the PRISMA statement. PLoS Med 6:e1000097. https://doi.org/10.1371/journ al.pmed.1000097

11. Lazarus G, Rinaldi I (2020) Immune checkpoint inhibitors in the treatment of cancer patients with COVID-19 infection: a systematic review protocol. PROSPERO CRD42020202142. https://www.crd.york.ac.uk/PROSPERO/display_record.php? RecordID=202142. Accessed 18 Sept 2020

12. World Health Organization (2020) Clinical management of COVID-19: interim guidance, 27 May 2020. World Health Organization. https://apps.who.int/iris/handle/10665/332196. Accessed 15 Aug 2020

13. Szumilas M (2010) Explaining odds ratios. J Can Acad Child Adolesc Psychiatry 19:227-229

14. Higgins JPT, Eldridge S, Li T (2020) Chapter 23: including variants on randomized trials. In: Higgins J, Thomas J, Chandler J et al (eds) Cochrane handbook for systematic reviews of interventions version 6.1. Cochrane. https://training.cochrane. org/handbook/archive/v6.1. Accessed 8 Mar 2021

15. Hayden JA, Côté P, Bombardier C (2006) Evaluation of the quality of prognosis studies in systematic reviews. Ann Intern Med 144:427-437. https://doi.org/10.7326/0003-4819-144-6200603210-00010

16. R Core Team (2020) R: a language and environment for statistical computing. R Foundation for Statistical Computing, Vienna

17. Balduzzi S, Rücker G, Schwarzer G (2019) How to perform a meta-analysis with R: a practical tutorial. Evid Based Ment Health 22:153-160. https://doi.org/10.1136/ebmen tal-2019-300117

18. DerSimonian R, Laird N (1986) Meta-analysis in clinical trials. Control Clin Trials 7:177-188. https://doi.org/10.1016/01972456(86)90046-2

19. Higgins JPT, Thomas J, Chandler J et al (2019) Cochrane handbook for systematic reviews of interventions version 6.0 (updated July 2019). Cochrane. https://training.cochrane.org/handbook/ archive/v6. Accessed 15 Aug 2020

20. Peters JL, Sutton AJ, Jones DR et al (2008) Contour-enhanced meta-analysis funnel plots help distinguish publication bias from other causes of asymmetry. J Clin Epidemiol 61:991-996. https:// doi.org/10.1016/j.jclinepi.2007.11.010 
21. Egger M, Smith GD, Schneider M, Minder C (1997) Bias in metaanalysis detected by a simple, graphical test. BMJ 315:629-634. https://doi.org/10.1136/bmj.315.7109.629

22. Begg CB, Mazumdar M (1994) Operating characteristics of a rank correlationtTest for publication bias. Biometrics 50:1088-1101

23. Huguet A, Hayden JA, Stinson J et al (2013) Judging the quality of evidence in reviews of prognostic factor research: adapting the GRADE framework. Syst Rev 2:71. https://doi.org/10.1186/ 2046-4053-2-71

24. Assaad S, Avrillon V, Fournier ML et al (2020) High mortality rate in cancer patients with symptoms of COVID-19 with or without detectable SARS-COV-2 on RT-PCR. Eur J Cancer 135:251-259. https://doi.org/10.1016/j.ejca.2020.05.028

25. Gonzalez-Cao M, Antonazas-Basa M, Puertolas T, et al (2020) Cancer immunotherapy does not increase the risk of death by COVID-19 in melanoma patients. medRxiv 2020.05.19.20106971. https://doi.org/10.1101/2020.05.19.20106971

26. Lee LYW, Cazier J-B, Angelis V et al (2020) COVID-19 mortality in patients with cancer on chemotherapy or other anticancer treatments: a prospective cohort study. Lancet 395:1919-1926. https://doi.org/10.1016/S0140-6736(20)31173-9

27. Pinato DJ, Zambelli A, Aguilar-Company J et al (2020) Clinical portrait of the SARS-CoV-2 epidemic in European patients with cancer. Cancer Discov 10:1465-1474. https://doi.org/10.1158/ 2159-8290.cd-20-0773

28. Yarza R, Bover M, Paredes D et al (2020) SARS-CoV-2 infection in cancer patients undergoing active treatment: analysis of clinical features and predictive factors for severe respiratory failure and death. Eur J Cancer 135:242-250. https://doi.org/10.1016/j.ejca. 2020.06.001

29. Lara OD, O'Cearbhaill RE, Smith MJ et al (2020) COVID-19 outcomes of patients with gynecologic cancer in New York City. Cancer 126:4294-4303. https://doi.org/10.1002/cncr.33084

30. Luo J, Rizvi H, Egger JV et al (2020) Impact of PD-1 blockade on severity of COVID-19 in patients with lung cancers. Cancer Discov 10:1121-1128. https://doi.org/10.1158/2159-8290. CD-20-0596

31. Robilotti EV, Babady NE, Mead PA et al (2020) Determinants of COVID-19 disease severity in patients with cancer. Nat Med 26:1218-1223. https://doi.org/10.1038/s41591-020-0979-0

32. Tyan K, Bui A-T, Giobbie-Hurder A et al (2020) 481 Impact of COVID-19 on cancer patients receiving immune checkpoint inhibitors. J Immunother Cancer 8:A515-A516. https://doi.org/ 10.1136/jitc-2020-sitc2020.0481

33. Dai M, Liu D, Liu M et al (2020) Patients with cancer appear more vulnerable to SARS-CoV-2: a multicenter study during the COVID-19 outbreak. Cancer Discov 10:783-791. https://doi.org/ 10.1158/2159-8290.CD-20-0422

34. Garassino MC, Whisenant JG, Huang L-C et al (2020) COVID-19 in patients with thoracic malignancies (TERAVOLT): first results of an international, registry-based, cohort study. Lancet Oncol 21:914-922. https://doi.org/10.1016/S1470-2045(20)30314-4

35. Sterne JAC, Sutton AJ, Ioannidis JPA et al (2011) Recommendations for examining and interpreting funnel plot asymmetry in meta-analyses of randomised controlled trials. BMJ. https://doi. org/10.1136/bmj.d4002

36. Kuderer NM, Choueiri TK, Shah DP et al (2020) Clinical impact of COVID-19 on patients with cancer (CCC19): a cohort study. Lancet 395:1907-1918. https://doi.org/10.1016/S0140-6736(20) 31187-9

37. Tian J, Yuan X, Xiao J et al (2020) Clinical characteristics and risk factors associated with COVID-19 disease severity in patients with cancer in Wuhan, China: a multicentre, retrospective, cohort study. Lancet Oncol 21:893-903. https://doi.org/10.1016/S14702045(20)30309-0

38. Taur Y (2020) Systematic review data and elucidation of findings [personal communication]. Email to: Y Taur. 19 September 2020
39. Remon J, Mezquita L, Corral J et al (2018) Immune-related adverse events with immune checkpoint inhibitors in thoracic malignancies: focusing on non-small cell lung cancer patients. J Thorac Dis 10:1516-1533. https://doi.org/10.21037/jtd.2017.12. 52

40. Russano M, Citarella F, Napolitano A et al (2020) COVID-19 pneumonia and immune-related pneumonitis: critical issues on differential diagnosis, potential interactions, and management. Expert Opin Biol Ther 20:959-964. https://doi.org/10.1080/14712 598.2020.1789097

41. Ürün Y, Hussain SA, Bakouny Z et al (2020) Survey of the impact of COVID-19 on oncologists' decision making in cancer. JCO Glob Oncol 6:1248-1257. https://doi.org/10.1200/GO.20.00300

42. Tagliamento M, Spagnolo F, Poggio F et al (2020) Italian survey on managing immune checkpoint inhibitors in oncology during COVID-19 outbreak. Eur J Clin Invest 50:e13315. https://doi.org/ 10.1111/eci.13315

43. NHS England (2020) Clinical guide for the management of non-coronavirus patients requiring acute treatment : Cancer (23 March 2020 version 2). https://www.england.nhs.uk/coronavirus/ wp-content/uploads/sites/52/2020/03/specialty-guide-acute-treat ment-cancer-23-march-2020.pdf. Accessed 20 Aug 2020

44. Bersanelli M (2020) Controversies about COVID-19 and anticancer treatment with immune checkpoint inhibitors. Immunotherapy $12: 269-273$

45. Gatto L, Franceschi E, Di NV, Brandes AA (2020) Potential protective and therapeutic role of immune checkpoint inhibitors against viral infections and COVID-19. Immunotherapy. https:// doi.org/10.2217/imt-2020-0109

46. Citarella F, Russano M, Pantano F et al (2020) Facing SARSCoV-2 outbreak in immunotherapy era. Future Oncol 16:14751485. https://doi.org/10.2217/fon-2020-0340

47. Gambichler T, Reuther J, Scheel CH, Becker JC (2020) On the use of immune checkpoint inhibitors in patients with viral infections including COVID-19. J Immunother Cancer. https://doi.org/10. 1136/jitc-2020-001145

48. Di Cosimo S, Malfettone A, Pérez-García JM et al (2020) Immune checkpoint inhibitors: a physiology-driven approach to the treatment of coronavirus disease 2019. Eur J Cancer 135:62-65. https://doi.org/10.1016/j.ejca.2020.05.026

49. Finelli C (2020) Obesity, COVID-19 and immunotherapy: the complex relationship! Immunotherapy. 12:1105-1109. https:// doi.org/10.2217/imt-2020-0178

50. Sehgal K, Costa DB, Rangachari D (2020) Extended-interval dosing strategy of immune checkpoint inhibitors in lung cancer: will it outlast the COVID-19 pandemic? Front Oncol 10:1193. https:// doi.org/10.3389/fonc. 2020.01193

51. Liu C, Zhao Y, Okwan-Duodu D et al (2020) COVID-19 in cancer patients: risk, clinical features, and management. Cancer Biol Med 17:519-527

52. Quaglino P, Fava P, Brizio M et al (2020) Metastatic melanoma treatment with checkpoint inhibitors in the COVID-19 era: experience from an Italian Skin Cancer Unit. J Eur Acad Dermatol Venereol 34:1395-1396. https://doi.org/10.1111/jdv.16586

53. Erratum: department of error (2020) Lancet 396:534. https://doi. org/10.1016/S0140-6736(20)31758-X

54. Correction: impact of PD-1 blockade on severity of COVID-19 in patients with lung cancers (2021) Cancer Discov 11:520-520. https://doi.org/10.1158/2159-8290.cd-20-1818

Publisher's Note Springer Nature remains neutral with regard to jurisdictional claims in published maps and institutional affiliations. 